Trauma Berufskrankh $2015 \cdot$ [Suppl 2]:

17:283-286

DOI 10.1007/s10039-015-0002-z

Online publiziert: 24. März 2015

๑) Springer-Verlag Berlin Heidelberg 2015

\author{
M. Bonner \\ Berufsgenossenschaft der Bauwirtschaft (BG BAU), Berlin, Deutschland
}

\section{Reha-Management: Erwartungen und Erfahrungen}

Kaum ein anderer Begriff hat die gesetzliche Unfallversicherung in den letzten Jahren wohl so geprägt, wie der des Reha-Managements. Von Beginn an wurden hohe Erwartungen in das Konzept, dessen primäres Ziel eine optimale Gestaltung der Heilverfahrens- und Wiedereingliederungsprozesse ist, gesetzt. Nach den bisherigen Erkenntnissen der Unfallversicherungs(UV)-Träger werden diese Erwartungen insgesamt in hohem Maße erfüllt. Strukturell und aus Prozesssicht werden allerdings in einzelnen Punkten durchaus Optimierungsbedarfe gesehen. Dieser Beitrag soll einen Überblick zu den Erwartungen und zum aktuellen Erfahrungsstand der UV-Träger vermitteln.

\section{Entwicklung des Reha-Managements}

Um die Erwartungen an das Reha-Management besser einzuordnen zu können, ist ein Blick auf die Bearbeitung komplexer Fälle in der Vergangenheit hilfreich. In der Regel wurden diese Fälle gemeinschaftlich von klassischen Sachbearbeitern sowie der Berufshilfe betreut. Während die Sachbearbeitung im Innendienst für die Fallbearbeitung zuständig war, kümmerte sich die Berufshilfe vor Ort vornehmlich bei Fragen der beruflichen Wiedereingliederung - persönlich um die Versicherten. Nicht selten existierten nur unzureichende Regelungen zur Zusammenarbeit. Erschwert wurde diese zusätzlich, wenn beide Rollen in unterschiedlichen Organisationseinheiten agierten und es dadurch zu internen Abstimmungsproblemen kam.
Insgesamt bestand hinsichtlich der Steuerung und Überwachung der Heilverfahren auf mehreren Ebenen Optimierungsbedarf. Folgende Problemfelder konnten identifiziert werden:

- Ermittlungen wurden vorwiegend schriftlich durchgeführt. Hierdurch ging wertvolle Zeit verloren.

- Die einzelnen Phasen der Rehabilitation sowie der beruflichen und sozialen Wiedereingliederung wurden nicht stringent geplant und durchgeführt.

- Die Reha-Phasen waren nur unzureichend aufeinander abgestimmt.

- Die Folgen eines Unfalls für die berufliche und soziale Perspektive wurden zu spät erkannt.

Ende der 1990er Jahre begannen die Diskussionen über eine Optimierung der klassischen Prozesse. Besonders hervorzuheben sind in diesem Zusammenhang die Aktivitäten der Verwaltungsberufsgenossenschaft, die ihre neuen Abläufe in einer Veröffentlichung aus dem Jahr 2002 vorstellte [4]. In dem Beitrag finden sich bereits zahlreiche Gedanken, die später auch Eingang in den Handlungsleitfaden der Deutschen Gesetzlichen Unfallversicherung (DGUV) finden sollten.

In den folgenden Jahren installierten weitere UV-Träger das Reha-Management. Wenngleich die Prozesse und Strukturen in den einzelnen Häusern unterschiedlich ausgestaltet waren, bestand doch Konsens hinsichtlich der Zielsetzung der Aktivitäten bzw. der Erwartungen an das Reha-Management.

Erste Bestrebungen zur Formulierung trägerübergreifender Standards mündeten im Jahr 2008 in die „Eckpunkte für ein gemeinsames Reha-Management in der gesetzlichen Unfallversicherung " ${ }^{\text {[2] }}$. Dieses Papier, in dem auch die zwischenzeitlich gemachten Erfahrungen der einzelnen UV-Träger berücksichtigt wurden, kann heute als erster Meilenstein auf dem Weg zum Reha-Management angesehen werden.

Eine Fortentwicklung und weitere Konkretisierung wurden im Jahr 2010 durch den Handlungsleitfaden der DGUV zum Reha-Management [3] erreicht. Hier wurden die Rahmenbedingungen und Mindeststandards für das Reha-Management unter Beteiligung aller UV-Träger festgelegt. Dabei wurden aus den bisherigen Erfahrungen Maßnahmen abgeleitet, die sich in der Praxis der Träger bewährt hatten. Kernelemente des RehaManagements - und damit die entscheidenden Weiterentwicklungen zum bisherigen Verfahren - sind demnach:

- Planen und Begleiten der Rehabilitation sowie der Leistungen zur Teilhabe

- Steuern mithilfe eines Reha-Plans

- Einbinden aller Beteiligten

- Orientieren an der Internationalen Klassifikation der Funktionsfähigkeit, Behinderung und Gesundheit (ICF)

\section{Erwartungen}

Der Handlungsleitfaden zum Reha-Management legt das Fundament für eine weitgehend einheitliche Bearbeitung komplexer Fälle in der gesetzlichen Unfallversicherung. Mit der Formulierung eines gemeinsamen Rahmens gehen auch einheitliche Erwartungen an den Nutzen des Reha-Managements bei allen UVTrägern einher. Übergeordnet wird zum 


\section{BG BAU}

\section{Erwartungen an das Reha-Management}

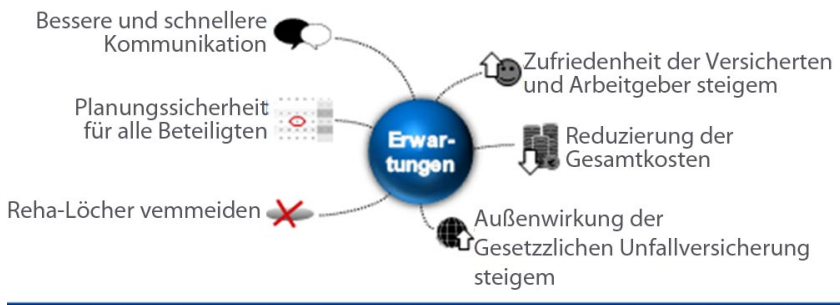

32. Unfallmedizinische Tagung $\quad$ Martin Bonner-BG BAU- $\quad 21 . / 22.11 .2014 \quad$ Seite 8

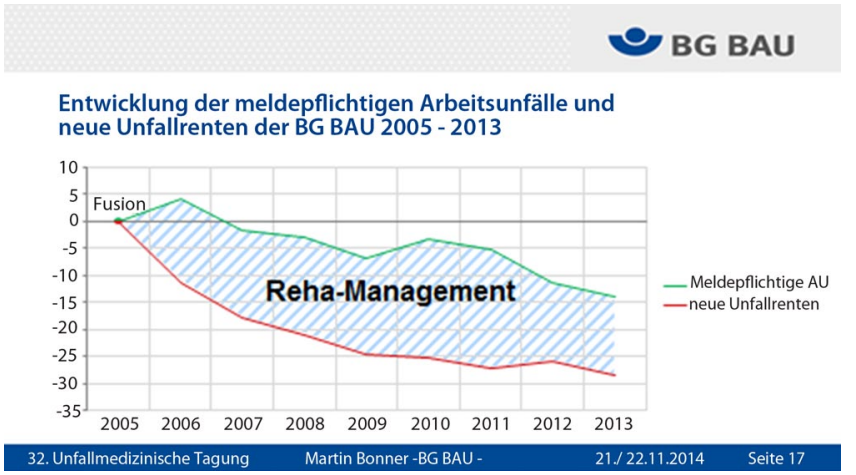

Abb. $2<$ Entwicklung der meldepflichtigen Arbeitsunfälle und neuen Unfallrenten der BG BAU 20052013

Wohl der Versicherten eine Steigerung der Effektivität und Effizienz bei den Heilverfahren und der Wiedereingliederung Schwerstverletzter angestrebt. Dieses Ziel lässt sich auf eine Vielzahl von Teilerwartungen herunterbrechen $(\bullet$ Abb. 1$)$.

Die Kommunikation zwischen allen Beteiligten sollte verbessert werden. Dies galt auch für den internen Informationsfluss bei den UV-Trägern. Die Einführung des Reha-Plans wurde mit einer erhöhten Planungssicherheit für alle Beteiligten assoziiert. Die Betroffenen sollten wissen, welche konkreten Maßnahmen wann, wo und durch wen vorgesehen sind. Eine frühzeitige Kontaktaufnahme und enge Begleitung des Rehabilitations- und Wiedereingliederungsprozesses sollte der Vermeidung kontraproduktiver Stillstandsphasen, der „Reha-Löcher" dienen.

Als Konsequenz aus der intensiven Fallsteuerung wurden letztlich auch eine Erhöhung der Kundenzufriedenheit, die Reduzierung der Gesamtkosten sowie eine bessere Außenwirkung der gesetzlichen Unfallversicherung erwartet.
Abb. $1<$ Erwartungen an das Reha-Management

\section{Erfahrungen}

Ob sich diese - mitunter hohen - Erwartungen bereits realisiert haben, wird nachfolgend bewertet.

\section{Kommunikation}

Die UV-Träger haben ihre Organisation an die Reha-Management-Prozesse angepasst oder beschäftigen sich mit den notwendigen Veränderungen. Zwar existieren unter den UV-Trägern unterschiedliche Vorstellungen, allerdings scheinen sich im Wesentlichen zwei Modelle aktuell durchzusetzen: Entweder agiert der Reha-Manager sowohl im Innen- als auch im Außendienst oder ausschließlich vor Ort mit Unterstützung durch die interne Sachbearbeitung. In der Regel ist der Reha-Manager heute (mit-)verantwortlich für alle Phasen der Rehabilitation und Teilhabe. Insgesamt haben die organisatorischen Veränderungen zu deutlich effizienteren Kommunikationsflüssen geführt.

Das Erstellen und Fortschreiben des Reha-Plans hat sich als wichtige Grundlage für einen optimalen Verfahrensablauf bewährt und die Kommunikation mit al- len Beteiligten verbessert. Von besonderer Bedeutung sind in diesem Zusammenhang auch der Aufbau und die Pflege von Netzwerken aus Leistungserbringern und Leistungsträgern zu nennen. Hierdurch wird eine enge, beschleunigte und vertrauensvolle Interaktion in Gang gesetzt. Reha-Löcher können so vermieden und dem Grundsatz der gesetzlichen Unfallversicherung, Leistungen „aus einer Hand“ zu erbringen, kann optimal Rechnung getragen werden.

Eine besondere Rolle im Reha-Management kommt den behandelnden Ärzten zu. Problematisch gestaltet sich deren Einbindung in der Praxis dann, wenn die Planung der Rehabilitation an anderer Stelle erfolgt, notwendige Informationen nicht zeitgerecht geliefert werden und dadurch Irritationen entstehen. Immer wieder wird von den Behandlern zu Recht eine Optimierung der Kommunikation gefordert.

\section{Planungssicherheit}

Der Reha-Plan als Vereinbarung über den Ablauf der Rehabilitations- und Teilhabephasen schafft unbestritten die notwendige Planungssicherheit für die Versicherten und deren Angehörigen. Diese können nun besser disponieren als in der Vergangenheit. Zeitintervalle ohne Aktivitäten treten seltener auf.

Ein Ursprungsgedanke bestand allerdings auch darin, dem Unternehmer regelmäßig Informationen an die Hand zu geben, nach denen er die notwendigen betriebswirtschaftlichen Entscheidungen treffen kann. In der Regel wird der Arbeitgeber zwar frühzeitig zur Feststellung des Tätigkeitsprofils kontaktiert. Die weitere Beteiligung erfolgt dann zu oft jedoch erst wieder zum Abschluss des Verfahrens, etwa wenn eine Arbeitsbelastungserprobung erforderlich wird. Eine zukünftig engere Einbeziehung des Unternehmers wäre wünschenswert.

\section{Kundenzufriedenheit}

Für eine Bewertung der Kundenzufriedenheit liegen noch keine aussagekräftigen Untersuchungsergebnisse vor. Erste Erfahrungen von Reha-Managern sowie aus Kundenbefragungen einzelner 
UV-Träger deuten jedoch eindeutig auf einen positiven Effekt des Reha-Managements hin.

In einem Forschungsprojekt der DGUV soll nun im Rahmen einer Vollerhebung die Zufriedenheit der im RehaManagement betreuten Versicherten gemessen werden. Das Projekt startet im Jahr 2015 und erstreckt sich über einen Zeitraum von 2 Jahren.

\section{Wirtschaftlichkeit}

Zur Wirtschaftlichkeit des Reha-Managements liegen bisher keine Erkenntnisse vor, die strengen wissenschaftlichen Anforderungen genügen. Erste belastbare Ergebnisse liefert jedoch das Benchmarking-Projekt „Effektivität und Wirtschaftlichkeit der Fallsteuerung“. Untersucht wurde u. a. anhand verschiedener Diagnosen die Auswirkung einer Fallsteuerung im Sinne des Reha-Managements gegenüber einer herkömmlichen Bearbeitungsweise. Für Unterschenkelfrakturen konnte beispielsweise eine durchschnittliche Reduzierung der Arbeitsunfähigkeitsdauer um 80 Tage durch die Bearbeitung im Reha-Management festgestellt werden [1]. Die Ergebnisse des BenchmarkingProjekts lassen den Schluss zu, dass die Kosten für Rehabilitation und Entschädigung durch eine Steuerung im Reha-Management insgesamt gesenkt werden.

Verschiedene UV-Träger haben eigene Wirtschaftlichkeitsuntersuchungen angestellt. So belegen die Zahlen der BG BAU, dass nach Einführung eines einheitlichen Reha-Managementverfahrens im Jahr 2005 die neuen Unfallrenten im Vergleich zu den meldepflichtigen Unfällen überproportional zurückgingen. Diese Feststellung spricht dafür, dass durch die frühzeitige und enge Steuerung komplexer Fälle das Ausmaß der Unfallfolgen beeinflusst werden kann (• Abb. 2).

\section{Außenwirkung}

Vier Jahre nach Veröffentlichung des Handlungsleitfadens existiert kein einheitliches Reha-Management der UVTräger. Dies war jedoch auch nicht $\mathrm{zu}$ erwarten. In dem Leitfaden wurden die Mindeststandards und Rahmenbedingungen des Verfahrens festgelegt und zu

Trauma Berufskrankh 2015 · [Suppl 2]: 17:283-286 DOI 10.1007/s10039-015-0002-z

(c) Springer-Verlag Berlin Heidelberg 2015

\section{Bonner}

\section{Reha-Management: Erwartungen und Erfahrungen. Ein Überblick}

\section{Zusammenfassung}

Hintergrund. Schwerverletzte werden durch die gesetzliche Unfallversicherung im Rahmen eines Reha-Managements betreut. Fragestellung. Erwartungen an und Erfahrungen mit dem Reha-Management. Material und Methode. Rückblick auf die Entstehung des Reha-Managements, Vergleich zwischen Erwartungen und Erfahrungen, Bestimmung des Optimierungsbedarfs. Ergebnisse. Die Erwartungen an das RehaManagement wurden weitgehend erfüllt. Vereinzelt besteht weiterhin Optimierungsbedarf, der im Sinne eines dynamischen Prozesses umgesetzt werden muss.
Schlussfolgerungen. Die Durchführung des Reha-Managements lohnt sich für alle Beteiligten. Das Verfahren führt zu einer effektiveren und effizienteren Steuerung der Heilverfahren und Teilhabeleistungen. Die Zufriedenheit der Kunden wird gesteigert, die Gesamtkosten werden gesenkt und die Außendarstellung der gesetzlichen Unfallversicherung kann gestärkt werden.

Schlüsselwörter

Reha-Management · Arbeitsunfall · Unfallversicherung · Qualitätssicherung · Schwerverletzte

\section{Rehabilitation management: expectations and experiences. An overview}

\section{Abstract}

Background. Severely injured persons are supported by the statutory accident insurance through rehabilitation management. Objectives. Expectations and experiences with regard to rehabilitation management Methods. Review the development of rehabilitation management, compare expectations and experiences and identify room for improvement.

Results. The expectations with regard to rehabilitation management were largely fulfilled. In some cases there is still room for improvement, which has to be addressed in a dynamic process.
Conclusion. Rehabilitation management is worthwhile for everyone involved. The procedure results in a more effective and efficient supervision of treatment and participation. It hence allows customer satisfaction to be improved, total costs to be reduced and the reputation of the statutory accident insurance to be strengthened.

Keywords

Rehabilitation management · Occupational accident $\cdot$ Accident insurance $\cdot$ Quality assurance $\cdot$ Severely injured diesen besteht ein breiter Konsens. Die Ausgestaltung des Rahmens ist Aufgabe der einzelnen UV-Träger. Insgesamt ist es durch das weitgehend einheitliche Auftreten der UV-Träger und die Etablierung der „Marke“ Reha-Management zu einer verbesserten Außenwirkung der gesetzlichen Unfallversicherung gekommen.

\section{Ausblick}

Aufgrund der positiven Erfahrungen im Unfallbereich ist geplant, im Jahr 2015 auf Ebene der DGUV ein Reha-Management bei Berufskrankheiten einzuführen. Im Fokus stehen hier v. a. Krebserkrankungen und andere komplexe Fallgestaltungen, die einen besonderen Steuerungsbedarf haben.
Für das Reha-Management wurden 5 Kennzahlen definiert, anhand derer zukünftig eine Qualitätsmessung und -sicherung erfolgen soll. Die laufende Qualitätssicherung dient der Weiterentwicklung des Reha-Managements. Der Handlungsleitfaden wird zu Recht als „paper in progress" angesehen, da bei Vorliegen neuer Erkenntnisse die Prozesse anzupassen sind. Verantwortlich hierfür ist die Arbeitsgruppe Reha-Management der DGUV mit ihren Unterarbeitsgruppen. Die größte Herausforderung besteht aus Sicht des Autors darin, die Prozesse einfach zu gestalten und eine Überregulierung zu verhindern. Entscheidend muss immer sein, dass praktikable Lösungen im Interesse der Kunden gefunden werden. 


\section{Fazit}

- Der Handlungsleitfaden zum RehaManagement legt die Mindeststandards und Rahmenbedingungen für alle UV-Träger fest.

- Die Erwartungen an das Reha-Management wurden weitgehend erfüllt.

\section{Korrespondenzadresse}

M. Bonner
Berufsgenossenschaft der
Bauwirtschaft (BG BAU)
Hildegardstraße 29/30
10715 Berlin
Martin.Bonner@bgbau.de

\section{Einhaltung ethischer Richtlinien}

Interessenkonflikt. M. Bonner gibt an, dass kein Interessenkonflikt besteht.

Dieser Beitrag beinhaltet keine Studien an Menschen oder Tieren.

The supplement containing this article is not sponsored by industry.

\section{Literatur}

1. Botti J et al (2013) Benchmarking Projekt abgeschlossen - Heilverfahrenssteuerung lohnt sich. DGUV Forum 7-8:36-39

2. DGUV (2008) Rundschreiben Reha 020/2008 vom 13.03.2008 Eckpunkte für ein gemeinsames RehaManagement in der gesetzlichen Unfallversicherung. DGUV, Berlin

3. DGUV (2010) Das Reha-Management der Deutschen Gesetzlichen Unfallversicherung - Handlungsleitfaden vom 13.09.2010. http://www.dguv. de/medien/inhalt/rehabilitation/documents/ handlungsleitfaden.pdf. Zugegriffen: 11. Dez. 2014

4. Froese E (2002) Dialogisches Reha-Management Ausdruck eines zeitgemäßen Selbstverständnisses eines Unfallversicherungsträgers. Die BG 3:

122-127 